\title{
Pseudo-Riemannian manifolds with recurrent spinor fields
}

\author{
Anton S. Galaev
}

November 1, 2018

\begin{abstract}
The existence of a recurrent spinor field on a pseudo-Riemannian spin manifold $(M, g)$ is closely related to the existence of a parallel 1-dimensional complex subbundle of the spinor bundle of $(M, g)$. We characterize the following simply connected pseudo-Riemannian manifolds admitting such subbundles in terms of their holonomy algebras: Riemannian manifolds; Lorentzian manifolds; pseudo-Riemannian manifolds with irreducible holonomy algebras; pseudo-Riemannian manifolds of neutral signature admitting two complementary parallel isotropic distributions.
\end{abstract}

Keywords: Pseudo-Riemannian manifold, recurrent spinor field, holonomy algebra.

\section{1 introduction}

Let $(M, g)$ be a pseudo-Riemannian spin manifold of signature $(r, s)$ and $S$ the corresponding complex spinor bundle with the induced connection $\nabla^{S}$. A spinor field $s \in \Gamma(S)$ is called recurrent if

$$
\nabla_{X}^{S} s=\theta(X) s
$$

for all vector fields $X \in \Gamma(T M)$, here $\theta$ is a complex-valued 1-form. If $\theta=0$, then $s$ is a parallel spinor field. For a recurrent spinor field $s$ there exist locally defined non-vanishing function $f$ such that $f s$ is parallel if and only if $d \theta=0$. If $M$ is simply connected, then such function exists globally.

The study of Riemannian spin manifolds carrying parallel spinor fields was initiated by N. Hitchin [1], and then by Th. Friedrich [2]. M. Wang characterized simply connected Riemannian spin manifolds admitting parallel spinor field in terms of their holonomy groups [3]. A similar result was obtained by Th. Leistner for Lorentzian manifolds [4, 5], by H. Baum and I. Kath for pseudo-Riemannian manifolds with irreducible holonomy groups [6], and by A. Ikemakhen in the case of pseudo-Riemannian manifolds of neutral signature $(n, n)$ admitting two complementary parallel isotropic distributions [7].

In [2], Th. Friedrich considered Equation (11) on a Riemannian spin manifold with $\theta$ being a real-valued 1-form. He proved that this equation implies that the Ricci tensor is zero and $d \theta=0$. Below we will see that this statement does not hold for Lorentzian manifolds. Example 
1 from [2] provides a solution $s$ to (11) with $\theta=\mathrm{i} \omega, d \omega \neq 0$ for a real-valued 1-form $\omega$ on the compact Riemannian manifold $(M, g)$ being the product of the non-flat torus $T^{2}$ and the circle $S^{1}$. In fact the recurrent spinor field $s$ comes from a locally defined recurrent spinor field on the non-Ricci-flat Kähler manifold $T^{2}$ existing by Theorem 1 below.

The spinor bundle $S$ of $(M, g)$ admits a parallel 1-dimensional complex subbundle if and only if $(M, g)$ admits non-vanishing recurrent spinor fields in a neighborhood of each point such that these fields are proportional on the intersections of the domains of their definitions. In the present paper we study some classes of pseudo-Riemannian manifolds $(M, g)$ whose spinor bundles admit parallel 1-dimensional complex subbundles.

In Section 3 we prove that if the spinor bundle of a locally indecomposable Riemannian spin manifold $(M, g)$ admits a parallel 1-dimensional complex subbundle, then either $(M, g)$ is a non-Ricci-flat Kählerian manifold, or it admits a non-zero parallel spinor field. Moreover, the spinor bundle of any locally indecomposable non-Ricci-flat Kählerian spin manifold admits exactly two parallel 1-dimensional complex subbundles. Next, any simply connected complete Riemannian spin manifold with not irreducible holonomy algebra whose spinor bundle $S$ admits a parallel 1-dimensional complex subbundle and does not admit any non-zero parallel spinor field is a product of a non-Ricci flat Kählerian spin manifold and of a Riemannian spin manifold admitting a parallel spinor field.

In Section 4 we prove that the spinor bundle of an $n+2$-dimensional simply connected locally indecomposable Lorentzian spin manifold $(M, g)$ admits a parallel 1-dimensional complex subbundle if and only if $(M, g)$ admits a parallel distribution of isotropic lines, i.e. its holonomy algebra $\mathfrak{g}$ is contained in the parabolic subalgebra

$$
\mathfrak{s i m}(n)=(\mathbb{R} \oplus \mathfrak{s o}(n)) \ltimes \mathbb{R}^{n} \subset \mathfrak{s o}(1, n+1),
$$

and the subalgebra $\mathfrak{h}=\operatorname{pr}_{\mathfrak{s o}(n)} \mathfrak{g} \subset \mathfrak{s o}(n)$ preserves a 1-dimensional complex subspace in the spinor module $\Delta_{n}$.

In Section 5 for pseudo-Riemannian spin manifolds with irreducible holonomy algebras we prove the same statements as for locally indecomposable Riemannian manifolds in Section 3 .

In Section 6] we prove that the spinor bundle of any simply connected pseudo-Riemannian spin manifold of neutral signature $(n, n)$, admitting two complementary parallel isotropic distributions, admits a parallel 1-dimensional complex subbundle.

In Section 7 we discuss the relation between recurrent spinor fields and parallel spinor fields of $\operatorname{spin}^{C}$-bundles that has been studied by A. Moroianu [8] and A. Ikemakhen [9, 10].

Acknowledgments. The author is thankful to Helga Baum for proposing this problem and the discussions on this topic.

\section{Preliminaries}

Spinor representations. Let us fix some standard denotation. Let $\mathbb{R}^{r, s}$ be a pseudo-Euclidean space with the metric $g$ of signature $(r, s)$ ( $r$ denotes the number of minuses). Let $\left(\mathcal{C} l_{r, s}, \cdot\right)$ be 
the corresponding Clifford algebra and $\mathbb{C l}_{r, s}=\mathcal{C} l_{r, s} \otimes \mathbb{C}$ be its complexification. The last algebra can be represented as an matrix algebra in the following way. Consider the basis

$$
\left(u(\epsilon)=\frac{\sqrt{2}}{2}\left(\begin{array}{c}
1 \\
-\epsilon \mathrm{i}
\end{array}\right), \epsilon= \pm 1\right)
$$

of $\mathbb{C}^{2}$. Define the following isomorphisms of $\mathbb{C}^{2}$ :

$$
E=\mathrm{id}, \quad T=\left(\begin{array}{cc}
0 & -\mathrm{i} \\
\mathrm{i} & 0
\end{array}\right), \quad U=\left(\begin{array}{cc}
\mathrm{i} & 0 \\
0 & -\mathrm{i}
\end{array}\right), \quad V=\left(\begin{array}{cc}
0 & \mathrm{i} \\
\mathrm{i} & 0
\end{array}\right) .
$$

It holds

$$
\begin{gathered}
T^{2}=-V^{2}=-U^{2}=E, \quad U T=-\mathrm{i} V, \quad V T=i U, \quad U V=-\mathrm{i} T, \\
T u(\epsilon)=-\epsilon u(\epsilon), \quad U u(\epsilon)=\mathrm{i} u(-\epsilon), \quad T u(\epsilon)=\epsilon u(-\epsilon) .
\end{gathered}
$$

Let $n=r+s$. A basis $e_{1}, \ldots, e_{n}$ of $\mathbb{R}^{r, s}$ is called orthonormal if $g\left(e_{i}, e_{j}\right)=k_{i} \delta_{i j}$, where $k_{i}=-1$ if $1 \leq i \leq r$, and $k_{i}=1$ if $r+1 \leq i \leq n$. Let us fix such basis. For an integer $m$ denote by $\mathbb{C}(m)$ the algebra of the complex square matrices of order $m$. Define the following isomorphisms:

$1)$ if $n$ is even, then define $\Phi_{r, s}: \mathbb{C} l_{r, s} \rightarrow \mathbb{C}\left(2^{\frac{n}{2}}\right)$ by

$$
\begin{gathered}
\Phi_{r, s}\left(e_{2 k-1}\right)=\tau_{2 k-1} E \otimes \cdots \otimes E \otimes U \otimes \underbrace{T \otimes \cdots \otimes T}_{(k-1)-\text { times }}, \\
\Phi_{r, s}\left(e_{2 k}\right)=\tau_{2 k} E \otimes \cdots \otimes E \otimes V \otimes \underbrace{T \otimes \cdots \otimes T}_{(k-1) \text {-times }},
\end{gathered}
$$

where $1 \leq k \leq \frac{n}{2}, \tau_{i}=\mathrm{i}$ if $1 \leq i \leq r$, and $\tau_{i}=1$ if $r+1 \leq i \leq n$;

2) if $n$ is odd, then define $\Phi_{r, s}: \mathbb{C} l_{r, s} \rightarrow \mathbb{C}\left(2^{\frac{n-1}{2}}\right) \oplus \mathbb{C}\left(2^{\frac{n-1}{2}}\right)$ by

$$
\begin{aligned}
& \Phi_{r, s}\left(e_{k}\right)=\left(\Phi_{r, s-1}\left(e_{k}\right), \Phi_{r, s-1}\left(e_{k}\right)\right), \quad k=1, \ldots, n-1, \\
& \Phi_{r, s}\left(e_{n}\right)=(T \otimes \cdots \otimes T,-\mathrm{i} T \otimes \cdots \otimes T) .
\end{aligned}
$$

The obtained representation space $\Delta_{r, s}=\mathbb{C}^{2^{\left[\frac{n}{2}\right]}}$ is called the spinor module. We write $A \cdot s=$ $\Phi_{r, s}(A) s$ for all $A \in \mathbb{C} l_{r, s}, s \in \Delta_{r, s}$. We will consider the following basis of $\Delta_{r, s}$ :

$$
\left(u\left(\epsilon_{k}, \ldots, \epsilon_{1}\right)=u\left(\epsilon_{k}\right) \otimes \cdots \otimes u\left(\epsilon_{1}\right) \mid \epsilon_{i}= \pm 1\right) .
$$

Recall that the Lie algebra $\mathfrak{s p i n}(r, s)$ of the Lie group $\operatorname{Spin}(r, s)$ can be embedded into $\mathbb{C} l_{r, s}$ in the following way:

$$
\mathfrak{s p i n}(r, s)=\operatorname{span}\left\{e_{i} \cdot e_{j} \mid 1 \leq i<j \leq n\right\} .
$$

The Lie algebra $\mathfrak{s o}(r, s)$ can be identified with the space of bivectors $\Lambda^{2} \mathbb{R}^{r, s}$ in such a way that

$$
(x \wedge y) z=g(x, z) y-g(y, z) x, \quad x, y, z \in \mathbb{R}^{r, s} .
$$

There is the isomorphism

$$
\lambda_{*}: \mathfrak{s o}(r, s) \rightarrow \mathfrak{s p i n}(r, s), \quad \lambda_{*}(x \wedge y)=x \cdot y
$$


The obtained representation of $\mathfrak{s o}(r, s)$ in $\Delta_{r, s}$ is irreducible if $n$ is odd, and this representation splits into the direct some of two irreducible modules

$$
\Delta_{r, s}^{ \pm}=\operatorname{span}\left\{u\left(\epsilon_{k}, \ldots, \epsilon_{1}\right) \mid \epsilon_{1}=\cdots=\epsilon_{k}= \pm 1\right\}
$$

if $n$ is even.

Holonomy algebras. Let $(M, g)$ be a pseudo-Riemannian manifold of signature $(r, s)$ and $\nabla$ the Levi-Civita connection on $(M, g)$. Fix a point $x \in M$. The tangent space $T_{x} M$ can be identified with the pseudo-Euclidean space $\mathbb{R}^{r, s}$. Then the holonomy algebra $\mathfrak{h}_{x} \subset \mathfrak{s o}\left(T_{x} M, g_{x}\right)$ (i.e. the Lie algebra of the holonomy group) of $(M, g)$ at the point $x$ is identified with a subalgebra $\mathfrak{h} \subset \mathfrak{s o}(r, s)$. If $M$ is simply connected, then the holonomy algebra and the holonomy group unequally define each other, and we prefer to speak about the holonomy algebra. If $(M, g)$ is a spin manifold, then it admits a spinor bundle $S$. The fiber $S_{x}$ can be identified with the spinor module $\Delta_{r, s}$. The Levi-Civita connection $\nabla$ defines a connection $\nabla^{S}$ on the spinor bundle $S$. The holonomy algebra of this connection coincides with $\lambda_{*}(\mathfrak{h}) \subset \mathfrak{s p i n}(r, s)$. The fundamental property of the holonomy algebra says that if $(M, g)$ is simply connected, then $(M, g)$ admits a parallel vector field (spinor, distribution, and so on) if and only if $\mathfrak{h}$ annihilates a vector in $\mathbb{R}^{r, s}$ (annihilates a spinor in $\Delta_{r, s}$, preserves a vector subspace in $\mathbb{R}^{r, s}$, and so on).

\section{Riemannian manifolds}

Theorem 1 Let $(M, g)$ be a locally indecomposable $n$-dimensional simply connected Riemannian spin manifold. Then its spinor bundle $S$ admits a parallel 1-dimensional complex subbundle if and only if either the holonomy algebra $\mathfrak{h} \subset \mathfrak{s o}(n)$ of $(M, g)$ is one of $\mathfrak{u}\left(\frac{n}{2}\right), \mathfrak{s u}\left(\frac{n}{2}\right), \mathfrak{s p}\left(\frac{n}{4}\right)$, $G_{2} \subset \mathfrak{s o}(7), \mathfrak{s p i n}(7) \subset \mathfrak{s o}(8)$, or $(M, g)$ is a locally symmetric Kählerian manifold.

Proof. Recall that $S$ admits a parallel 1-dimensional complex subbundle if and only if the holonomy algebra $\mathfrak{h} \subset \mathfrak{s o}(n)$ preserves a 1-dimensional complex subspace $l$ of the spinor module $\Delta_{n}$. If $\mathfrak{h}$ annihilates $l$, then $(M, g)$ admits a non-zero parallel spinor field, and $\mathfrak{h}$ is one of $\mathfrak{s u}\left(\frac{n}{2}\right)$, $\mathfrak{s p}\left(\frac{n}{4}\right), G_{2}, \mathfrak{s p i n}(7)$ [3]. Suppose that $\mathfrak{h}$ does not annihilate $l$. Since $\mathfrak{h} \subset \mathfrak{s o}(n)$, it is a compact Lie algebra. It is clear that the only compact Lie algebra admitting a non-trivial exact 2dimensional real representation is $\mathfrak{s o}(2)$. This shows that $\mathfrak{h}$ contains a 1-dimensional center, consequently, $\mathfrak{h}$ is contained in $\mathfrak{u}\left(\frac{n}{2}\right)$, i.e. $(M, g)$ is Kählerian. Since $\mathfrak{s u}\left(\frac{n}{2}\right)$ annihilates two complex 1-dimensional subspaces of $\Delta_{n}, \mathfrak{u}\left(\frac{n}{2}\right)$ preserves these two subspaces and it does not annihilate them. This shows that the spinor bundle $S$ of a Kählerian manifold admits at least two parallel 1-dimensional complex subbundles. And it admits exactly two such subbundles if $\mathfrak{h}=\mathfrak{u}\left(\frac{n}{2}\right)$.

Corollary 1 Let $(M, g)$ be a simply connected Riemannian spin manifold with irreducible holonomy algebra and without non-zero parallel spinor fields. Then the spinor bundle $S$ admits a parallel 1-dimensional complex subbundle if and only if $(M, g)$ is a Kählerian manifold and it is not Ricci-flat. 
Proof. The corollary follows from the fact that simply connected Riemannian manifolds with the holonomy algebras $\mathfrak{s u}\left(\frac{n}{2}\right), \mathfrak{s p}\left(\frac{n}{4}\right), G_{2}, \mathfrak{s p \mathfrak { i n }}(7)$ do admit non-zero parallel spinor fields.

Corollary 2 Let $(M, g)$ be a simply connected complete Riemannian spin manifold without non-zero parallel spinor fields and with not irreducible holonomy algebra. Then its spinor bundle $S$ admits a parallel 1-dimensional complex subbundle if and only if $(M, g)$ is a direct product of a Kählerian not Ricci-flat spin manifold and of a Riemannian spin manifold with a non-zero parallel spinor field.

Proof. By the de Rham Theorem, $(M, g)$ can be decomposed as a direct product of indecomposable simply connected complete Riemannian manifolds and, probably, of a flat Riemannian manifold, see e.g. [11]. The Riemannian manifolds in this decomposition are automatically spin, and the spinor bundle $S$ of $(M, g)$ admits a parallel 1-dimensional complex subbundle if and only if this is the case for each manifold in the decomposition.

Theorem 2 Let $(M, g)$ be a locally indecomposable $n$-dimensional simply connected non-Ricciflat Kählerian spin manifold. Then its spinor bundle $S$ admits exactly two parallel 1-dimensional complex subbundles.

This theorem will follow from a more general Theorem 7 that will be proved below.

\section{Lorentzian manifolds}

In this section we consider Lorentzian manifolds $(M, g)$, i.e. pseudo-Riemannian manifolds of signature $(1, n+1), n \geq 0$. Holonomy algebras of Lorentzian spin manifold admitting non-zero parallel spinor fields are classified in [4, 5]. We suppose now that the spinor bundle of $(M, g)$ admits a parallel 1-dimensional complex subbundle and $(M, g)$ does not admit any parallel spinor.

Theorem 3 Let $(M, g)$ be a simply connected complete Lorentzian spin manifold. Suppose that $(M, g)$ does not admit a parallel spinor. Then its spinor bundle $S$ admits a parallel 1dimensional complex subbundle if and only if one of the following conditions holds:

1) $(M, g)$ is a direct product of $\left(\mathbb{R},-(d t)^{2}\right)$ and of a Riemannian spin manifold $(N, h)$ such that the spinor bundle of $(N, h)$ admits a parallel 1-dimensional complex subbundle and $(N, h)$ does not admit any non-zero parallel spinor field;

2) $(M, g)$ is a direct product of an indecomposable Lorentzian spin manifold and of Riemannian spin manifold $(N, h)$ such that the spinor bundles of both manifolds admit parallel 1-dimensional complex subbundles and at least one of these manifolds does not admit any non-zero parallel spinor field. 
Proof. The proof of this theorem follows directly from the Wu decomposition Theorem, the proof of a similar statement can be found in [4, 5].

Note that if $(M, g)$ admits a parallel spinor field, then it satisfies the analog of Theorem 3 with $(N, h)$ admitting a parallel spinor field [4, 5].

Now we may consider locally indecomposable Lorentzian manifolds $(M, g)$. Suppose that the spinor bundle of $(M, g)$ admits a parallel 1-dimensional complex subbundle $l$. Let $s \in \Gamma(l)$ be a local non-vanishing section of $l$. Let $p \in \Gamma(T M)$ be its Dirac current. Recall that $p$ is defined from the equality

$$
g(p, X)=-<X \cdot s, s>
$$

where $<,>$ is a Hermition product on $S$. We claim that $p$ is a recurrent vector field. Indeed, following the computations from [5], for any vector fields $X$ and $Y$ we get

$$
\begin{aligned}
g\left(\nabla_{Y} p, X\right) & =Y(g(p, X))-g\left(p, \nabla_{Y} X\right)=-\left(Y(<X \cdot s, s>)-<\nabla_{Y} X \cdot s, s>\right) \\
& =-\left(<\nabla_{Y} X \cdot s, s>+<X \cdot \nabla_{Y}^{S} s, s>+<X \cdot s, \nabla_{Y}^{S} s>-<\nabla_{Y} X \cdot s, s>\right) \\
& =-(\theta(Y)+\overline{\theta(Y)})<X \cdot s, s>=(\theta(Y)+\overline{\theta(Y)}) g(p, X) .
\end{aligned}
$$

We obtain

$$
\nabla_{Y} p=2 \operatorname{Re}(\theta)(Y) p
$$

Recall that in the Lorentzian signature the Dirac current satisfies $g(p, p) \leq 0$ and the zeros of $p$ coincide with the zeros of $s$. Since $s$ is non-vanishing and $p$ is recurrent, we see that either $g(p, p)<0$, or $g(p, p)=0$. In the first case the manifold is decomposable. Thus we get that $p$ is an isotropic recurrent vector field. We conclude that $(M, g)$ admits a parallel distribution of isotropic lines. The holonomy algebras of such manifolds are classified [12, 13, 14, 11. We recall this classification now.

Let $(M, g)$ be a locally indecomposable Lorentzian manifold of dimension $n+2$. Suppose that $(M, g)$ admits a parallel distribution of isotropic lines. The tangent space to $(M, g)$ at any point can be identified with the Minkowski space $\mathbb{R}^{1, n+1}$. We fix a basis $p, e_{1}, \ldots, e_{n}, q$ of $\mathbb{R}^{1, n+1}$ such that the only non-zero values of the metric are $g(p, q)=g(q, p)=1$ and $g\left(e_{i}, e_{i}\right)=1$. We will denote by $\mathbb{R}^{n} \subset \mathbb{R}^{1, n+1}$ the Euclidean subspace spanned by the vectors $e_{1}, \ldots, e_{n}$. Suppose that $\mathbb{R} p$ corresponds to the parallel distribution of isotropic lines. Denote by $\mathfrak{s i m}(n)$ the subalgebra of $\mathfrak{s o}(1, n+1)$ that preserves the isotropic line $\mathbb{R} p$. The Lie algebra $\mathfrak{s i m}(n)$ can be identified with the following matrix algebra:

$$
\mathfrak{s i m}(n)=\left\{\left(\begin{array}{ccc}
a & X^{t} & 0 \\
0 & A & -X \\
0 & 0 & -a
\end{array}\right) \mid a \in \mathbb{R}, A \in \mathfrak{s o}(n), X \in \mathbb{R}^{n}\right\} .
$$

The above matrix can be identified with the triple $(a, A, X)$. We get the decomposition

$$
\mathfrak{s i m}(n)=(\mathbb{R} \oplus \mathfrak{s o}(n)) \ltimes \mathbb{R}^{n},
$$

which means that $\mathbb{R} \oplus \mathfrak{s o}(n) \subset \mathfrak{s i m}(n)$ is a subalgebra and $\mathbb{R}^{n} \subset \mathfrak{s i m}(n)$ is an ideal, and the Lie brackets of $\mathbb{R} \oplus \mathfrak{s o}(n)$ with $\mathbb{R}^{n}$ are given by the standard representation of $\mathbb{R} \oplus \mathfrak{s o}(n)$ in $\mathbb{R}^{n}$. The holonomy algebra $\mathfrak{g}$ of $(M, g)$ is contained in $\mathfrak{s i m}(n)$. Any subalgebra $\mathfrak{h} \subset \mathfrak{s o}(n)$ can be decomposed into the direct sum $\mathfrak{h}=\mathfrak{h}^{\prime} \oplus \mathfrak{z}(\mathfrak{h})$, where $\mathfrak{h}^{\prime}=[\mathfrak{h}, \mathfrak{h}]$ and $\mathfrak{z}(\mathfrak{h})$ is the center of $\mathfrak{h}$. 
Theorem 4 A subalgebra $\mathfrak{g} \subset \mathfrak{s i m}(n)$ is the holonomy algebra of a locally indecomposable Lorentzian manifold if and only if it is conjugated to one of the following subalgebras:

type $1 . \mathfrak{g}^{1, \mathfrak{h}}=(\mathbb{R} \oplus \mathfrak{h}) \ltimes \mathbb{R}^{n}$, where $\mathfrak{h} \subset \mathfrak{s o}(n)$ is the holonomy algebra of a Riemannian manifold;

type 2. $\mathfrak{g}^{2, \mathfrak{h}}=\mathfrak{h} \ltimes \mathbb{R}^{n}$, where $\mathfrak{h} \subset \mathfrak{s o}(n)$ is the holonomy algebra of a Riemannian manifold;

type 3. $\mathfrak{g}^{3, \mathfrak{h}, \varphi}=\{(\varphi(A), A, 0) \mid A \in \mathfrak{h}\} \ltimes \mathbb{R}^{n}$, where $\mathfrak{h} \subset \mathfrak{s o}(n)$ is the holonomy algebra of a Riemannian manifold with $\mathfrak{z}(\mathfrak{h}) \neq\{0\}$, and $\varphi: \mathfrak{h} \rightarrow \mathbb{R}$ is a non-zero linear map with $\left.\varphi\right|_{\mathfrak{h}^{\prime}}=0$

type 4. $\mathfrak{g}^{4, \mathfrak{h}, m, \psi}=\left\{(0, A, X+\psi(A)) \mid A \in \mathfrak{h}, X \in \mathbb{R}^{m}\right\}$, where $0<m<n$ is an integer, $\mathfrak{h} \subset \mathfrak{s o}(m)$ is the holonomy algebra of a Riemannian manifold with $\operatorname{dim} \mathfrak{z}(\mathfrak{h}) \geq n-m$, and $\psi: \mathfrak{h} \rightarrow \mathbb{R}^{n-m}$ is a surjective linear map with $\left.\psi\right|_{\mathfrak{h}^{\prime}}=0$.

Recall that for the subalgebra $\mathfrak{h} \subset \mathfrak{s o}(n)$ there is an orthogonal decomposition

$$
\mathbb{R}^{n}=\mathbb{R}^{n_{1}} \oplus \cdots \oplus \mathbb{R}^{n_{s}} \oplus \mathbb{R}^{n_{s+1}}
$$

and the corresponding decomposition into the direct sum of ideals

$$
\mathfrak{h}=\mathfrak{h}_{1} \oplus \cdots \oplus \mathfrak{h}_{s} \oplus\{0\}
$$

such that $\mathfrak{h}$ annihilates $\mathbb{R}^{n_{s+1}}, \mathfrak{h}_{i}\left(\mathbb{R}^{n_{j}}\right)=0$ for $i \neq j$, and $\mathfrak{h}_{i} \subset \mathfrak{s o}\left(n_{i}\right)$ is an irreducible subalgebra for $1 \leq i \leq s$. Moreover, the subalgebras $\mathfrak{h}_{i} \subset \mathfrak{s o}\left(n_{i}\right)$ are the holonomy algebras of Riemannian manifolds.

In [4, 5] it is proved that $(M, g)$ admits a non-zero parallel spinor field if and only if $\mathfrak{g}=\mathfrak{g}^{2, \mathfrak{h}}=$ $\mathfrak{h} \ltimes \mathbb{R}^{n}$ and in the decomposition (5) of $\mathfrak{h} \subset \mathfrak{s o}(n)$ each subalgebra $\mathfrak{h}_{i} \subset \mathfrak{s o}\left(n_{i}\right)$ coincides with one of the Lie algebras $\mathfrak{s u}\left(\frac{n_{i}}{2}\right), \mathfrak{s p}\left(\frac{n_{i}}{4}\right), G_{2} \subset \mathfrak{s o}(7), \mathfrak{s p i n}(7) \subset \mathfrak{s o}(8)$.

Theorem 5 Let $(M, g)$ be a simply connected locally indecomposable $(n+2)$-dimensional Lorentzian spin manifold. Then its spinor bundle $S$ admits a parallel 1-dimensional complex subbundle if and only if $(M, g)$ admits a parallel distribution of isotropic lines (i.e. its holonomy algebra $\mathfrak{g}$ is contained in $\mathfrak{s i m}(n)$ ), and in the decomposition (15) of $\mathfrak{h}=\operatorname{pr}_{\mathfrak{s o}(n)} \mathfrak{g}$ each subalgebra $\mathfrak{h}_{i} \subset \mathfrak{s o}\left(n_{i}\right)$ coincides with one of the Lie algebras $\mathfrak{u}\left(\frac{n_{i}}{2}\right), \mathfrak{s u}\left(\frac{n_{i}}{2}\right), \mathfrak{s p}\left(\frac{n_{i}}{4}\right), G_{2}, \mathfrak{s p i n}(7)$, or with the holonomy algebra of an indecomposable Kählerian symmetric space. The number of parallel 1-dimensional complex subbundles of $S$ equals to the number of 1-dimensional complex subspaces of $\Delta_{n}$ preserved by $\mathfrak{h}$.

Proof. It is enough to find for each Lie algebra $\mathfrak{g}$ from Theorem 4 all 1-dimensional complex invariant submodules of $\Delta_{1, n+1}$.

Under the identification $\mathfrak{s o}(1, n+1) \simeq \Lambda^{2} \mathbb{R}^{1, n+1}$, an element $(a, A, X) \in \mathfrak{s i m}(n)$ corresponds to the bivector

$$
-a p \wedge q+A-p \wedge X
$$


Recall that

$$
\Delta_{1, n+1} \simeq \Delta_{n} \otimes \Delta_{1,1}, \quad \Delta_{1,1} \simeq \mathbb{C}^{2} .
$$

Consider the vectors $e_{-}=\frac{\sqrt{2}}{2}(p-q)$ and $e_{+}=\frac{\sqrt{2}}{2}(p+q)$ and the orthonormal basis $e_{-}, e_{+}, e_{1}, \ldots, e_{n}$ of $\mathbb{R}^{1, n+1}$. Note that $p=\frac{\sqrt{2}}{2}\left(e_{-}+e_{+}\right)$and $q=\frac{\sqrt{2}}{2}\left(e_{-}-e_{+}\right)$.

Let us find all complex 1-dimensional $p \wedge \mathbb{R}^{n}$-invariant submodules of $\Delta_{1, n+1}$. Let $w \in \Delta_{1, n+1}$ be a spinor such that $\left(p \wedge e_{i}\right) \cdot w=c_{i} w, c_{i} \in \mathbb{C}$, for all $i=1, \ldots, n$. We may write

$$
w=w_{+} \otimes u(1)+w_{-} \otimes u(-1),
$$

where $w_{ \pm} \in \Delta_{n}$. Using (2), (3) and the computations from [4, 5] it is easy to get that

$$
\left(e_{i} \wedge p\right) \cdot w=\frac{\sqrt{2}}{2} e_{i} \cdot\left(e_{-}+e_{+}\right) \cdot w=\sqrt{2}\left(e_{i} \cdot w_{-}\right) \otimes u(1) .
$$

Hence the equalities $\left(p \wedge e_{i}\right) \cdot w=c_{i} w$ imply $c_{i}=0, e_{i} \cdot w_{-}=0$. Since the vectors $e_{i}$ act in $\Delta_{n}$ as isomorphisms, we get $w_{-}=0$. Thus, $w=w_{+} \otimes u(1)$, and all complex 1-dimensional $p \wedge \mathbb{R}^{n}$-invariant submodules of $\Delta_{1, n+1}$ are contained in $\Delta_{n} \otimes u(1)$. Moreover, this is a trivial $p \wedge \mathbb{R}^{n}$-module. The same statement holds for $p \wedge \mathbb{R}^{m}$ in the case of the Lie algebra $\mathfrak{g}^{4, \mathfrak{h}, m, \psi}$. Next,

$$
(p \wedge q) \cdot\left(w_{+} \otimes u(1)\right)=2 w_{+} \otimes u(1), \quad A\left(w_{+} \otimes u(1)\right)=A\left(w_{+}\right) \otimes u(1)
$$

for all $w_{+} \in \Delta_{n}$ and $A \in \mathfrak{s o}(n)$. This shows that all $\mathfrak{g}$-invariant 1-dimensional subspaces of $\Delta_{1, n+1}$ are of the form $l \otimes u(1)$, where $l \subset \Delta_{n}$ is an $\mathfrak{h}$-invariant 1-dimensional subspace. The theorem is true.

Examples of analytical Lorentzian manifolds with each possible holonomy algebra, in particular, with the holonomy algebras as in Theorem 5 can be obtained using the construction from [14].

Let us consider one example. Let $(M, g)$ be a simply connected Lorentzian spin manifold with the holonomy algebra $\mathfrak{g}=(\mathbb{R} \oplus \mathfrak{h}) \ltimes \mathbb{R}^{n}$, where $\mathfrak{h} \subset \mathfrak{s o}(n)$ is the holonomy algebra of a Riemannian manifold carrying a non-zero parallel spinor field. By Theorem 5 , on $(M, g)$ locally exists a recurrent spinor field $s$ (restricting the consideration to a local subset of $(M, g)$ with the same holonomy algebra, we may assume that $s$ is defined globally). By the assumption on $\mathfrak{h}$ and by the proof of Theorem 5 , the corresponding 1-form $\theta$ must be real-valued. By the fact that the manifolds with the holonomy algebra $\mathfrak{g}$ do not admit any non-zero parallel vector field, and by (4), $d \theta \neq 0$. This shows that the statement of Theorem 1 from [2] mentioned in Introduction does not hold for Lorentzian manifolds.

\section{Pseudo-Riemannian manifolds with irreducible holon- omy algebras}

Theorem 6 Let $(M, g)$ be a simply connected pseudo-Riemannian spin manifold of signature $(r, s)$ with an irreducible holonomy algebra $\mathfrak{h} \subset \mathfrak{s o}(r, s)$. Then its spinor bundle $S$ admits a parallel 1-dimensional complex subbundle if and only if either the holonomy algebra $\mathfrak{h}$ is one of $\mathfrak{u}\left(\frac{r}{2}, \frac{s}{2}\right), \mathfrak{s u}\left(\frac{r}{2}, \frac{s}{2}\right), \mathfrak{s p}\left(\frac{r}{4}, \frac{s}{2}\right), G_{2} \subset \mathfrak{s o}(7), G_{2(2)}^{*} \subset \mathfrak{s o}(3,4), G_{2}^{\mathbb{C}} \subset \mathfrak{s o}(7,7), \mathfrak{s p i n}(7) \subset \mathfrak{s o}(8)$, $\mathfrak{s p i n}(3,4) \subset \mathfrak{s o}(4,4), \mathfrak{s p i n}(7, \mathbb{C}) \subset \mathfrak{s o}(8,8)$, or $(M, g)$ is a locally symmetric pseudo-Kählerian manifold. 
Proof. Recall that irreducible holonomy algebras of simply connected pseudo-Riemannian manifolds $(M, g)$ admitting non-zero parallel spinor fields are exhausted by $\mathfrak{s u}\left(\frac{r}{2}, \frac{s}{2}\right), \mathfrak{s p}\left(\frac{r}{4}, \frac{s}{2}\right)$, $G_{2} \subset \mathfrak{s o}(7), G_{2(2)}^{*} \subset \mathfrak{s o}(3,4), G_{2}^{\mathbb{C}} \subset \mathfrak{s o}(7,7), \mathfrak{s p i n}(7) \subset \mathfrak{s o}(8), \mathfrak{s p i n}(3,4) \subset \mathfrak{s o}(4,4), \mathfrak{s p i n}(7, \mathbb{C}) \subset$ $\mathfrak{s o}(8,8)$ [6]. From [15] it follows that manifolds with each of these holonomy algebras are Ricci-flat.

Suppose that $(M, g)$ is not Ricci-flat and its spinor bundle $S$ admits a parallel 1-dimensional complex subbundle $l$. Let $s$ be a local section of $l$ defined at a point $y \in M$. Equation (1) easily implies

$$
R_{y}^{S}(X, Y) s_{y}=(d \theta)_{y}(X, Y) s_{y}
$$

for all $X, Y \in T_{y} M$. Here $R^{S}$ is the curvature tensor of the connection $\nabla^{S}$. This equation shows that $R_{y}^{S}(X, Y)$ acts on $l_{y}$ as a multiplications by complex numbers. This and the AmbroseSinger Theorem imply that the holonomy algebra $\mathfrak{h}_{x}, x \in M$, of the manifold $(M, g)$ acts on $l_{x}$ by multiplications by complex numbers and this action is non-trivial. Since $\mathfrak{h} \subset \mathfrak{s o}(r, s)$ is irreducible, it is a reductive Lie algebra. We get that $\mathfrak{h}$ contains a commutative ideal. By the Schur Lemma, this ideal is generated a the complex structure $J \in \mathfrak{u}\left(\frac{r}{2}, \frac{s}{2}\right)$. Consequently, $\mathfrak{h} \subset \mathfrak{u}\left(\frac{r}{2}, \frac{s}{2}\right)$. Since $\mathfrak{s u}\left(\frac{r}{2}, \frac{s}{2}\right)$ annihilates two 1-dimensional complex subspaces in $\Delta_{r, s}[6], \mathfrak{u}\left(\frac{r}{2}, \frac{s}{2}\right)$ preserves these subspaces. Consequently the spinor bundle of each pseudo-Kählerian manifold admits at least two parallel 1-dimensional complex subbundles.

Corollary 3 Let $(M, g)$ be a simply connected pseudo-Riemannian spin manifold with irreducible holonomy algebra and without non-zero parallel spinor fields. Then the spinor bundle $S$ admits a parallel 1-dimensional complex subbundle if and only if $(M, g)$ is a pseudo-Kählerian manifold and it is not Ricci-flat.

Theorem 7 Let $(M, g)$ be a simply connected not Ricci-flat pseudo-Kählerian spin manifold with an irreducible holonomy algebra. Then its spinor bundle $S$ admits exactly two parallel 1-dimensional complex subbundles.

Proof. Let $(M, g)$ be a simply connected not Ricci-flat pseudo-Riemannian spin manifold with an irreducible holonomy algebra. Suppose that the spinor bundle $S$ admits a parallel 1-dimensional complex subbundle $l$. Let $s$ be a local non-vanishing section of $l$, then $s$ is a recurrent spinor field. In the proof of Theorem 6 we have seen that $\mathfrak{h}$ contains a 1-dimensional commutative ideal generated by the complex structure $J \in \mathfrak{u}\left(\frac{r}{2}, \frac{s}{2}\right)$ and in Equality (6) $R_{x}(X, Y)$ acts on $s_{x}$ as its projection on $\mathbb{R} J \subset \mathfrak{h}_{x}$. Consequently Equality (6) implies that $(d \theta(X, Y))^{2}=$ $-a(X, Y)^{2}, a(X, Y) \in \mathbb{R}$, that is $d \theta=1 \omega$ for a real-valued 2-form $\omega$ on $M$.

Now we follow the ideas form [8] and [9]. The computations similar to [8, Lem. 3.1] and [2, Th. 2] show the equality

$$
\operatorname{Ric}(X) \cdot s=\mathrm{i}(X\lrcorner \omega) \cdot s
$$

for all vector fields $X$. Here Ric is the Ricci operator. Consider the distributions $T$ and $E$ defined in the following way:

$$
T_{x}=\left\{X \in T_{x} M \mid X \cdot s_{x}=0\right\}
$$




$$
E_{x}=\left\{X \in T_{x} M \mid \exists Y \in T_{x} M, X \cdot s_{x}=\mathrm{i} Y \cdot s_{x}\right\} .
$$

Since we may choose $s$ in a neighborhood of each point and any two such spinor fields are proportional on the intersections of the domains of their definitions, the distributions $T$ and $E$ are defined globally on $M$. We claim that the both distributions are parallel. Suppose that $X \in \Gamma(T)$, then for any local vector field $Z$ it holds

$$
\left(\nabla_{Z} X\right) \cdot s=\nabla_{Z}^{S}(X \cdot s)-X \cdot\left(\nabla_{Z}^{S} s\right)=0-X \cdot(\theta(Z) s)=-\theta(Z)(X \cdot s)=0,
$$

i.e. $T$ is parallel. Let $X \in \Gamma(E)$, then locally there exist vector field $Y$ such that $X \cdot s=\mathrm{i} Y \cdot s$. For any local vector field $Z$ it holds

$$
\left(\nabla_{Z} X\right) \cdot s=\nabla_{Z}^{S}(X \cdot s)-X \cdot\left(\nabla_{Z}^{S} s\right)=\nabla_{Z}^{S}(\mathrm{i} Y \cdot s)-X \cdot(\theta(Z) s)=\mathrm{i}\left(\nabla_{Z} Y+\theta(Z) Y-\theta(Z) Y\right) \cdot s=\mathrm{i}\left(\nabla_{Z} Y\right) \cdot s
$$

i.e. $E$ is parallel. Since Ric $\neq 0$ and $w=i d \theta \neq 0$, (7) shows that $T \neq T M$ and $E \neq 0$. Since the holonomy algebra of $(M, g)$ is irreducible, $T=0$ and $E=T M$. Thus for any local vector field $X$ there exists a unique vector field $Y$ such that $X \cdot s=\mathrm{i} Y \cdot s$. This defines an endomorphism $I$ of the tangent bundle such that

$$
X \cdot s=\mathrm{i} I(X) \cdot s
$$

Consequently,

$$
X \cdot s=\mathrm{i} I(X) \cdot s=-I^{2}(X) \cdot s, \quad\left(X+I^{2}(X)\right) \cdot s=0 .
$$

Since $T=0$, it holds $I^{2}(X)=-X$, i.e. $I$ is an almost complex structure on $M$. Now we show that $I$ is $g$-orthogonal. It holds

$$
\begin{array}{r}
2 g(I(X), I(Y)) s=I(X) \cdot I(Y) \cdot s+I(Y) \cdot I(X) \cdot s=-\mathrm{i}(I(X) \cdot Y \cdot s+I(Y) \cdot X \cdot s) \\
=-\mathrm{i}(2 g(I(X), Y) s-Y \cdot I(X) \cdot s+2 g(X, I(Y)) s-X \cdot I(Y) \cdot s) \\
=X \cdot Y \cdot s+Y \cdot X \cdot s-2 \mathrm{i}(g(I(X), Y)+g(X, I(Y))) s \\
=2 g(X, Y) s-2 \mathrm{i}(g(I(X), Y)+g(X, I(Y))) s .
\end{array}
$$

This implies, in particular, $g(I(X), I(Y))=g(X, Y)$. Next we claim that $I$ is parallel. Indeed, (8) implies for all vector fields $X$ and $Y$ the following:

$$
\nabla_{Y} X \cdot s+X \cdot \theta(Y) \cdot s=\mathrm{i} \nabla_{Y}(I X) \cdot s+\mathrm{i} I(X) \cdot \theta(Y) \cdot s .
$$

Hence,

$$
\theta(Y) X \cdot s=\mathrm{i} \nabla_{Y}(I X) \cdot s
$$

Using this and (8) applied to $\nabla_{Y} X$, we get

$$
0=\mathrm{i}\left(\nabla_{Y}(I X)-I\left(\nabla_{Y} X\right)\right) \cdot s
$$

This shows that $\nabla_{Y}(I X)-I\left(\nabla_{Y} X\right) \in \Gamma(T)$. Hence, $\nabla_{Y}(I X)-I\left(\nabla_{Y} X\right)=0$, i.e. $\nabla I=0$. Thus, $s$ defines a Kähler structure $I$. Note that this gives another proof of Theorem 6 .

Suppose that we have two recurrent spinor fields $s$ and $s_{1}$. These fields define two Kähler structures $I$ and $I_{1}$ satisfying (8) for $s$ and $s_{1}$, respectively. Since $I$ and $I_{1}$ are parallel, their values $I_{x}$ and $I_{1 x}$ at a point $x \in M$ commute with the holonomy algebra $\mathfrak{h}_{x} \subset \mathfrak{s o}\left(T_{x} M, g_{x}\right) \simeq$ 
$\mathfrak{s o}(r, s)$. Since $\mathfrak{h}_{x} \subset \mathfrak{s o}\left(T_{x} M, g_{x}\right)$ is irreducible, by the Schur Lemma, the centralizer of $\mathfrak{h}_{x}$ in $\mathfrak{s o}\left(T_{x} M, g_{x}\right)$ is isomorphic ether to $i \mathbb{R}$, or to $\mathfrak{s p}(1)$. In the last case, $\mathfrak{h}_{x}$ must be contained in $\mathfrak{s p}\left(\frac{r}{4}, \frac{s}{4}\right)$. This gives a contradiction, since the manifolds with such holonomy algebra are Ricci-flat. We conclude that $I_{x}=\alpha I_{1 x}$ for some $\alpha \in \mathbb{R}$. Since $I_{x}^{2}=I_{1 x}^{2}=-\mathrm{id}, \alpha= \pm 1$. Since the both tensor fields $I$ and $I_{1}$ are parallel, $I=\alpha I_{1}$.

Suppose that $I=I_{1}$. Let $\Omega$ be the pseudo-Kählerian form corresponding to $I$. Let $e_{1}, \ldots, e_{m}, I e_{1}, \ldots, I e_{m}$ $(r+s=n=2 m)$ be a local orthonormal basis of $\Gamma(T M)$, i.e. $g\left(e_{i}, e_{i}\right)=g\left(I e_{i}, I e_{i}\right)=k_{i}$, where $k_{i}=-1$ for $i=1, \ldots, \frac{r}{2}$, and $k_{i}=1$ for $i=\frac{r}{2}+1, \ldots, m$. Let $e^{1}, \ldots, e^{m},(I e)^{1}, \ldots,(I e)^{m}$ be the dual basis of $\Gamma(T M)$. Then

$$
\Omega=-\sum_{i=1}^{m} k_{i}(I e)^{i} \wedge e^{i}
$$

Using this and (8), we get

$$
\Omega \cdot s=-\sum_{i=1}^{m} k_{i}\left(I e_{i}\right) \cdot e_{i} \cdot s=-\mathrm{i} \sum_{i=1}^{m} k_{i}\left(I e_{i}\right) \cdot\left(I e_{i}\right) \cdot s=-\mathrm{i} m s .
$$

Similarly, $\Omega \cdot s_{1}=-\mathrm{i} m s_{1}$. Recall that the spinor bundle $S$ of a pseudo-Kählerian manifold admits the decomposition

$$
S=\sum_{k=0}^{m} S_{k}, \quad\left(S_{k}\right)_{x} \simeq \Lambda^{k} \mathbb{C}^{m}
$$

where $S_{k}$ is the eigenspace corresponding to the eigenvalue $(m-2 k)$ i of the operator of the Clifford multiplication by the form $\Omega$. We get that $s, s_{1} \in S_{0}$. Since $S_{0}$ is of rank $1, s$ and $s_{1}$ are proportional and they belong to the same 1-dimensional parallel subbundle of $S$. Above we have seen that $S$ admits at least two parallel 1-dimensional complex subbundles. We see now that it admits exactly two subbundles and the second one corresponds to the Kähler structure $-I$ (and coincides with $S_{m}$ that has dimension 1 ).

\section{Pseudo-Riemannian manifolds of neutral signature}

Theorem 8 Let $(M, g)$ be a simply connected pseudo-Riemannian spin manifolds of neutral signature $(n, n)$ admitting two complementary parallel isotropic distributions. Then the spinor bundle $S$ of $(M, g)$ admits a parallel 1-dimensional complex subbundle.

Proof. The tangent space of such manifold can be identified with the space $\mathbb{R}^{n, n}$ and it admits the decomposition $\mathbb{R}^{n, n}=W \oplus W_{1}$, where $W, W_{1} \subset \mathbb{R}^{n, n}$ are isotropic. The holonomy algebra of such manifold is of the form

$$
\widetilde{\mathfrak{h}}=\left\{A=\left(\begin{array}{cc}
B & 0 \\
0 & -B^{T}
\end{array}\right) \mid B \in \mathfrak{h}\right\}
$$

for a subalgebra $\mathfrak{h} \subset \mathfrak{g l}(n, \mathbb{R})$, which is the restriction of $\widetilde{\mathfrak{h}}$ to $W$. In [7] it is shown that $\widetilde{\mathfrak{h}}$ annihilates an element in $\Delta_{n, n}$ if and only if $\mathfrak{h} \subset \mathfrak{s l}(n, \mathbb{R})$. It is also proved that the above 
element $A \in \widetilde{\mathfrak{h}}$ acts in $\Delta_{n, n}$ as

$$
\frac{1}{2} n \cdot+\frac{1}{4} \sum_{i=1}^{n}\left(e_{i}^{*} \cdot A\left(e_{i}\right)-A\left(e_{i}^{*}\right) \cdot e_{i}\right)
$$

where $e_{1}, \ldots, e_{n}$ and $e_{1}^{*}, \ldots, e_{n}^{*}$ are bases of $W$ and $W_{1}$, respectively, such that $g\left(e_{i}, e_{j}^{*}\right)=\delta_{i j}$.

Hence $A_{0}=\left(\begin{array}{cc}E & 0 \\ 0 & -E\end{array}\right)$ acts in $\Delta_{n, n}$ as the multiplication by $n$. Since the Lie algebra $\widetilde{\mathfrak{s l}(n, \mathbb{R})}$ annihilates some non-zero elements of $\Delta_{n, n}$, the Lie algebra $\widetilde{\mathfrak{g l}(n, \mathbb{R})}=\widetilde{\mathfrak{s l}(n, \mathbb{R})} \oplus \mathbb{R} A_{0}$ preserves the corresponding lines in $\Delta_{n, n}$. This proves the theorem.

\section{$7 \quad$ Relation to parallel spinor fields of $\operatorname{spin}^{C}$ bundles}

Let $(M, g)$ be a simply connected pseudo-Riemannian spin manifold with the holonomy algebra $\mathfrak{h} \subset \mathfrak{s o}(p, q)$ and with the spin bundle $S$. Let $S^{C}$ be the $\operatorname{spin}^{C}$ bundle on $(M, g)$ given by a complex line bundle $L$ over $(M, g)$ with a connection 1 -form i $A$ and with the holonomy algebra $\mathfrak{h}_{L} \subset \mathrm{i} \mathbb{R}$. The holonomy algebra $\widehat{\mathfrak{h}}$ of the induced connection on $S^{C}$ is contained in $\lambda_{*}(\mathfrak{h}) \oplus \mathfrak{i} \mathbb{R} \subset \mathfrak{s p i n}(p, q) \oplus \mathfrak{i} \mathbb{R}$. In [9] it is proved that the existence of a non-zero parallel spinor field in $S^{C}$ is equivalent to the existence of a spinor $v \in \Delta_{p, q}$ such that $\xi \cdot v=$ it $v$ for all $(\xi, \mathrm{i} t) \in \widehat{\mathfrak{h}}$. Hence, the existence of a parallel spinor field in $S^{C}$ implies the existence of a parallel 1-dimensional complex subbundle of $S$. In general the converse statement is not true. For example, in the Lorentzian case the existence of a spinor field in $S^{C}$ implies the existence of a parallel vector field on $(M, g)$ [10], while we have seen above that there exist Lorentzian manifolds admitting recurrent spinor fields and no non-zero parallel vector fields.

\section{References}

[1] N. Hitchin, Harmonic spinors. Advances in Math. 14 (1974), 1-55.

[2] Th. Friedrich, Zur Existenz paralleler Spinorfelder über Riemannschen Mannigfaltigkeiten. Colloq. Math. 44 (1981), no. 2, 277-290.

[3] M. Wang, Parallel spinors and parallel forms. Ann. Global Anal. Geom. 7 (1989), no. 1, 59-68.

[4] Th. Leistner, Lorentzian manifolds with special holonomy and parallel spinors. Rend. Circ. Mat. Palermo (2) Suppl. 2002, no. 69, 131-159.

[5] Th. Leistner, Holonomy and parallel spinors in Lorentzian geometry. Berlin: Logos-Verlag; Humboldt-Universität zu Berlin, xii, 178 p. (2003).

[6] H. Baum, I. Kath, Parallel spinors and holonomy groups on pseudo-Riemannian spin manifolds. Ann. Global Anal. Geom. 17 (1999), no. 1, 1-17. 
[7] A. Ikemakhen, Groupes d'holonomie et spineurs parallèles sur les variétés pseudoriemanniennes complètement réductibles. C. R. Math. Acad. Sci. Paris 339 (2004), no. 3, 203-208.

[8] A. Moroianu, Parallel and Killing spinors on Spin $^{c}$ manifolds. Comm. Math. Phys. 187 (1997), no. 2, 417-427.

[9] A. Ikemakhen, Parallel spinors on pseudo-Riemannian Spin ${ }^{c}$ manifolds. J. Geom. Phys. 56 (2006), no. 9, 1473-1483.

[10] A. Ikemakhen, Parallel spinors on Lorentzian Spin ${ }^{c}$ manifolds. Differential Geom. Appl. 25 (2007), no. 3, 299-308.

[11] A. S. Galaev, Th. Leistner, Holonomy groups of Lorentzian manifolds: classification, examples, and applications. Recent developments in pseudo-Riemannian geometry, 53-96, ESI Lect. Math. Phys., Eur. Math. Soc., Zürich, 2008.

[12] L. Berard Bergery, A. Ikemakhen, On the Holonomy of Lorentzian Manifolds, Proceeding of symposia in pure math., volume 54 (1993), 27-40.

[13] Th. Leistner, On the classification of Lorentzian holonomy groups. J. Differential Geom. 76 (2007), no. 3, 423-484.

[14] A. S. Galaev, Metrics that realize all Lorentzian holonomy algebras, International Journal of Geometric Methods in Modern Physics, Vol. 3 (2006), Nos. 5-6, 1025-1045.

[15] S. Armstrong, Ricci-flat holonomy: a classification, J. Geom. Phys. 57 (2007), no. 6, 14571475. 